\title{
Coupled-Cavity Distributed-Resonance Photodetectors
}

\author{
I. Rendina*, F. Coppinger, B. Jalali, C. Lam, and E. Yablanovitch \\ Electrical Engineering Department, \\ UCLA, Box 951594, \\ Los Angeles, CA 90095-1594 \\ *National Council of Research of Italy, \\ IRECE, Via Diocleziano 328, \\ 80124 Napoli \\ Italy
}

\begin{abstract}
A new type of resonant enhanced photodetectors based on the one dimensional photonic bandgap is proposed. It can potentially overcome problems encountered in resonant-cavity-enhanced photodetectors. Resonating structures with very high quality factors and large free spectral ranges can be obtained by varying the optical parameters of the grating overlay without the presence of rigid design constraints. The use of coupled-cavities with distributed resonances is discussed. In particular, we demonstrate that the exploitation of cascaded structures allows resonant-enhanced parallel detection of wavelength Division Multiplexed (WDM) optical signals.
\end{abstract}

KEY WORDS: Resonant photodetector, photonic bandgap, silicon photonics, SiGe

\section{INTRODUCTION}

It is well known that performance of optoelectronic devices is enhanced by placing the active device structure inside a resonant cavity. In such structures, the presence of the cavity causes a large enhancement of resonant optical field at discrete wavelengths. In particular, this effect has been demonstrated in the realization of high speed photodetectors with thin absorption layers [1]. Both wavelength selectivity and high speed response make them ideal for wavelength division multiplexing (WDM) applications.

Usually, resonant cavity enhanced (RCE) photodetectors have a vertical, i.e., normal-to-thesurface, cavity realized by means of epitaxial growth techniques. In particular, the active absorbing region is made of a small bandgap semiconductor enclosed between two parallel Bragg mirrors. These structures are suitable for III-V compound semiconductor materials where a wide variety of lattice matched material 
with large refractive index difference are available. Photodetectors with high reflectivity mirrors ( $>95 \%)$ and wide bandwidth $(\sim 50 \mathrm{~nm})$ have been demonstrated. [2,3].

In recent years, much research effort has been devoted to the development of silicon-based optical receivers operating at wavelengths beyond the absorption edge of $\mathrm{Si}$ (viz., $>1.1 . \mu \mathrm{m}$ ). In particular, as an alternative to the complicated wafer bonding techniques necessary to bond InP-based photonic devices to $\mathrm{Si}$, epitaxial growth of narrow bandgap SiGe alloys on Si substrates has been successfully exploited [4-6]. However the limitation on film thickness imposed by the strain, and the small absorption coefficient of $\mathrm{SiGe}$ alloys limit the quantum efficiency of SiGe photodetectors. Application of the RCE concept to SiGe detectors is mainly limited by the allowable strain and refractive index contrast of the SiGe alloy at the wavelengths of interest for optical fiber communication (i.e., 1.3 and $1.55 \mu \mathrm{m}$ ) [1]. As a result, $\mathrm{Si} / \mathrm{SiGe}$ epitaxial multilayer mirrors of only $50 \%$ reflectivity at $1.5 \mu \mathrm{m}$ have been demonstrated [7]. The alternative

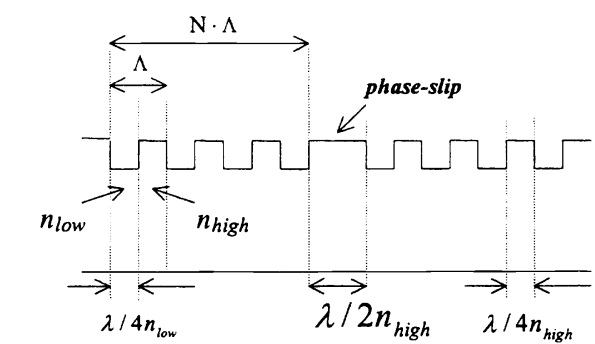

(a)

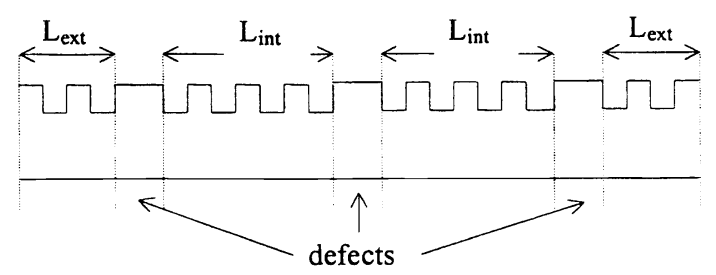

(b)

Figure 1: (a) Schematic of a single Photonic Bandgap cavity consiting of a grating with a defect "Phase slip" period. (b) Schematic of a cascaded photonic bandgap cavity, the grating contains several coupled defects.

approach to realize a resonant cavity using cleaved facets is not compatible with monolithic integration.

In this communication we propose a new class of resonant enhanced photodetectors based on waveguide structures with a high contrast Bragg grating overlay. The photonic bandgap (PBG) effect is exploited by incorporating single or cascaded phase-slip regions in the grating (see fig.1). Bragg gratings with a phase-slip section have been first and successfully developed for distributed feedback (DFB) lasers [8], and their use as optical filters in WDM systems has also been proposed [9-11]. PBG based structures allows resonant cavities with high quality factors and large free spectral range, That are suitable for integrated optics. 
The proposed detectors can significantly improve the saturation power of high-speed photodetectors which is limited due to the small absorption volume required for fast operation. In fact, in such devices a trade-off between effective absorption volume and bandwidths exists, and a solution to this problem seems to come from the exploitation of velocity-matched travelling-wave configuration [12-14]. The possibility of cascading several PBG cavities would make possible the realization of distributed RCE photodetectors with velocity-matched travelling-wave configuration. These devices would exhibit extremely fast operation, high saturation powers, wavelength selectivity, and high quantum efficiency.

The reflection and transmission spectrum of single and cascaded PBGs have been studied using a variety of theoretical approaches $[9,10,15]$. The matrix formalism [16,17], can predict not only the reflection and transmission spectra but also the internal electric field distribution throughout PBG structures [18]. We have used this technique to study single and cascaded PBGs for RCE photodetectors. We have designed resonant distributed cavities that allow the parallel detection of lightwave signals at different wavelengths for applications in WDM optical communication systems.

\section{THEORY}

A Bragg grating can be effectively represented as a multilayer structure made of layers with uniform refractive index. For this reason the calculation of the reflectivity and transmitivity of a PBG can be greatly simplified by using the "characteristic matrix" formalism according to which the whole structure is represented by a $2 \times 2$ matrix $(\underline{\underline{M}})$ relating the forward $(+)$ and backward $(-)$ propagating electric field amplitudes $\left(E^{\downarrow}\right)$ at the device input to that one at the output:

$$
\left[\begin{array}{l}
E_{\text {input }}^{+} \\
E_{\text {input }}^{-}
\end{array}\right]=\underline{\underline{M}} \cdot\left[\begin{array}{l}
E_{\text {output }}^{+} \\
E_{\text {output }}^{-}
\end{array}\right]
$$

The matrix $\underline{\underline{M}}$ is constructed by sequentially multiplying, from the input toward the output, the matrices representing the interfaces $(\underline{\underline{M}}$ interf.) between the adjacent layers and the matrices representing the layers themselves $(\underline{\underline{M}}$ layer $)$. If $n_{i}$ and $n_{i+1}$ are refractive indices of two adjacent layers, respectively,

$$
\underline{\underline{M}}_{\text {interf. }}=\frac{1}{2 n_{i}}\left[\begin{array}{ll}
n_{i}+n_{i+1} & n_{i}-n_{i+1} \\
n_{i}-n_{i+1} & n_{i}+n_{i+1}
\end{array}\right] .
$$

For the $i$-th layer:

$$
\underline{\underline{M}}_{\text {layer }}=\left[\begin{array}{cc}
e^{j \delta_{i}} & 0 \\
0 & e^{-j \delta_{i}}
\end{array}\right]
$$


$\delta_{i}$ is a phase coefficient defined by $\delta_{i}=2 \pi n_{i} l_{i} / \lambda$, where $\lambda$ is the wavelength in vacuum and $l_{i}$ is the layer length. The reflection and transmission coefficients characterizing the whole structure are respectively given by:

$$
\begin{gathered}
r=\left.\frac{E_{\text {input }}^{-}}{E_{\text {input }}^{+}}\right|_{E_{\text {ouput }}^{-}=0}=\frac{M(2,1)}{M(1,1)} \\
t=\left.\frac{E_{\text {output }}^{+}}{E_{\text {input }}^{+}}\right|_{E_{\text {ouput }}^{-}=0}=\frac{1}{M(1,1)}
\end{gathered}
$$

In addition to calculating the transmission and reflection coefficients for the entire structure, equation (1) can also be used for the determination of the electric field at any point (Z') inside the structure:

$$
\left[\begin{array}{l}
E^{+}\left(Z^{\prime}\right) \\
E^{-}\left(Z^{\prime}\right)
\end{array}\right]=\underline{\underline{M^{\prime}}} \cdot\left[\begin{array}{l}
t \\
0
\end{array}\right]
$$

where $\underline{\underline{M}}$ ' is the characteristic matrix representing the multilayer between $Z$ ' and the device output. From Eq. 6 the internal electric field at $Z$ ' is given by

$$
E\left(Z^{\prime}\right)=E^{+}\left(Z^{\prime}\right)+E^{-}\left(Z^{\prime}\right)=\frac{1}{M(1,1)} \cdot\left[M^{\prime}(1,1)+M^{\prime}(2,1)\right]
$$

Since the defect is exactly half a wavelength long, the beating term between $\mathrm{E}^{+}$and $\mathrm{E}^{-}$averages to zero over the length of the defect, resulting in an average power given by:

$$
I_{a v .}\left(Z^{\prime}\right)=\left|E^{+}\left(Z^{\prime}\right)\right|^{2}+\left|E^{-}\left(Z^{\prime}\right)\right|^{2}
$$

where the material dependent multiplying factor has been omitted. 


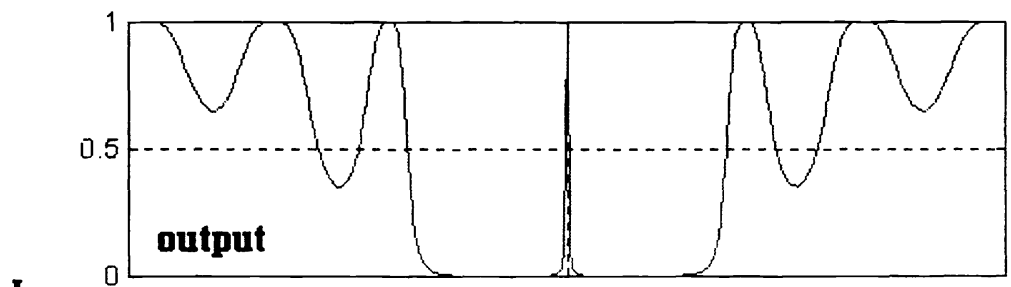

Iav.

(a.u.)

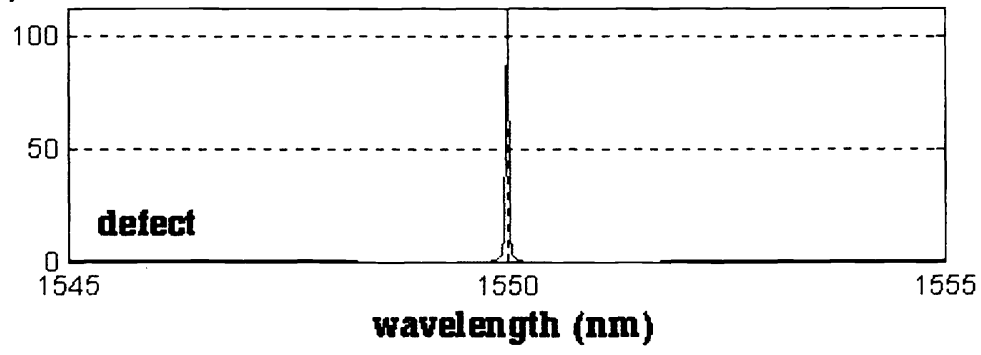

Figure 2: (Top) Average optical intensity at the output of the structure as a function of wavelength. (bottom) Optical intensity inside the defect.

\section{RESULTS AND DISCUSSION}

\section{III.1. Design fundamentals}

Figure 1 shows a single (a) and cascaded (b) PBG cavities. The main feature of the structure in Fig. 1a is that of exhibiting a narrow transmission peak centered at $\lambda_{B}$ (Bragg wavelength) in a stop band. This happens in analogy with the effect caused by a defect in an electronic crystal, viz., the appearance of an allowed energy state inside the crystal energy band-gap [19].

Figure 2 shows the average power intensity $\left(I_{a v}\right)$ at the device output and in the center of the defect as a function of the wavelength for the structure shown in Fig 1a. A unitary power intensity is assumed to impinge on the device input. Moreover, in all the simulation, the higher refractive index $n_{\text {high }}$ has been fixed to 3.7, and the Bragg wavelength to $1550 \mathrm{~nm}$. In particular, the plots in Fig. 2 refer to a PBG whose input and output sections have a number of periods $\mathrm{N}=1000$, and whose depth of the refractive index step ( $\Delta n=n_{\text {high }}-n_{\text {low }}$ ) is 0.01 . In general, increasing the number of periods results in a sharper resonance whereas increasing $\Delta \mathrm{n}$ mainly widens the stopband along with further narrowing of the resonance peak.

The transmission spectral characteristics of a PBG with $\mathrm{N}=1000$ and $\Delta n=0.01$ are comparable to that of a $200-\mu \mathrm{m}$-long Fabry-Perot cavity whose mirrors have reflectivity greater than $90 \%$. We have discussed the difficulty of realizing Si/SiGe Fabry-Perot cavity with such a high mirror reflectivity. PBGbased devices could overcome these problems. Moreover, the wide free-spectral-range characterizing 


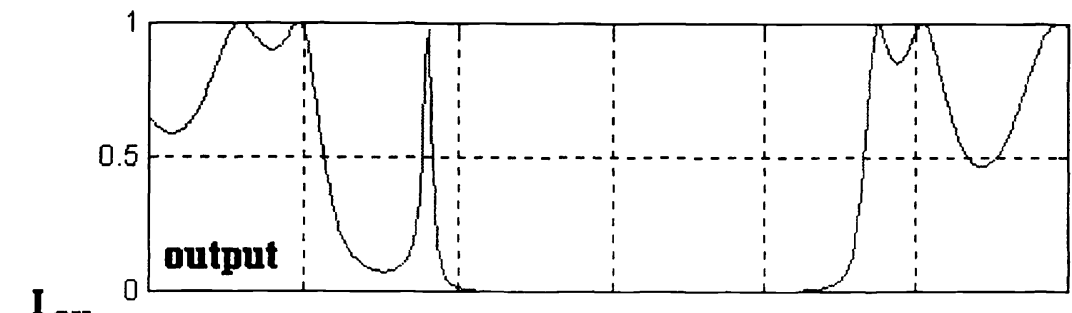

I av.

(a.u.)

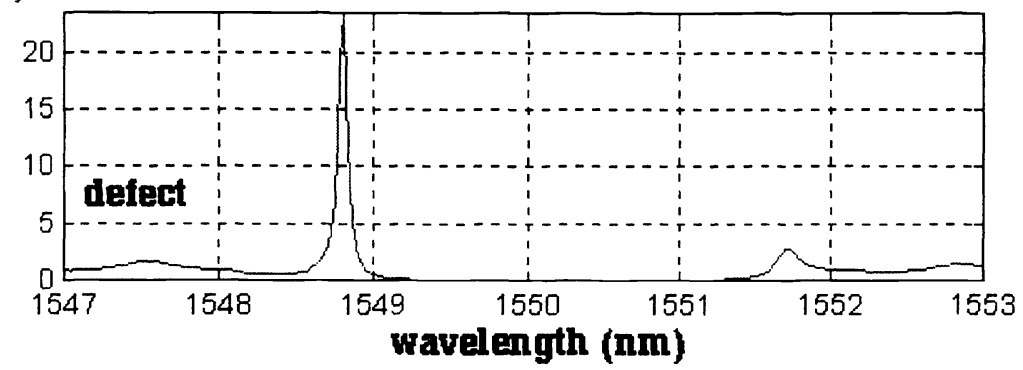

Figure 3: Optical intensity at the output (top), inside the defect (bottom), when the defect length is $\lambda_{b} / 3 n$

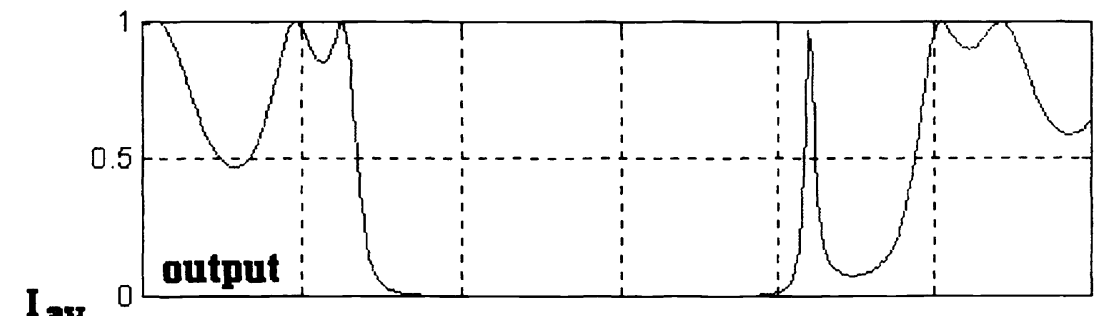

(a.u.)

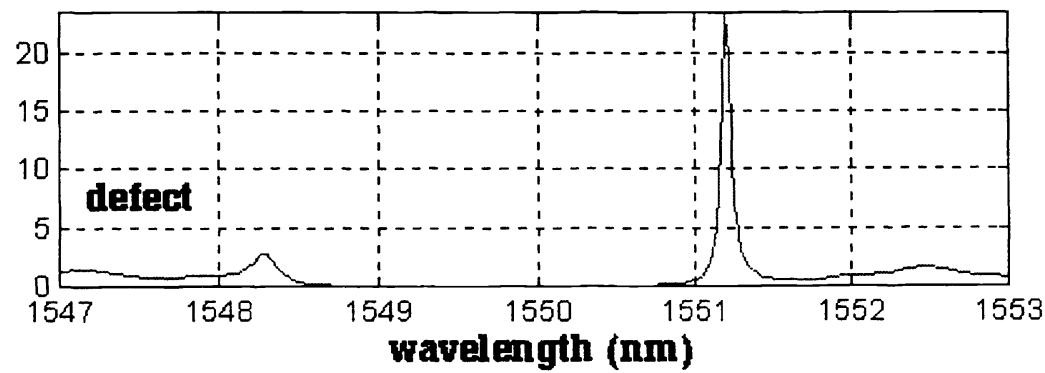

Figure 4: Optical Intensity at the (top), and inside the defect (bottom) when the defect length is $\lambda_{B} / 5 n$. 
the average power intensity spectrum calculated in the center of the defects of a simple PBG makes such structures highly desirable for the realization of wavelength-selective optical detectors for WDM systems.

If the defect has a length $\left(l_{D}\right)$ other than $\lambda_{B} / 2 n$, the transmission peak does not appear at the center of the stop-band. Figs. 3 and 4 show the response of PBGs with central defects of $\lambda_{B} / 3 n$ and $\lambda_{B} / 1.5 n$, respectively $(\mathrm{N}=1000$, and $\Delta n=0.01)$. This effect can be used to tune the resonant wavelength inside the stop-band. For instance, changing the optical length of the defect by means of the thermo-optic effect [20] can perform a wavelength tuning. A second way to tune the resonant wavelength is that of changing the optical length of both the defect and the mirrors. Changing, for instance, the temperature of the entire PBG structure can achieve this. In this case a shift of the stop-band along with the central transmission peak is obtained. The expression relating the variation of the Bragg resonant wavelength to the required change of the refractive index is $\Delta \lambda_{\mathrm{B}} / \lambda_{\mathrm{B}}=\Delta n / n$.

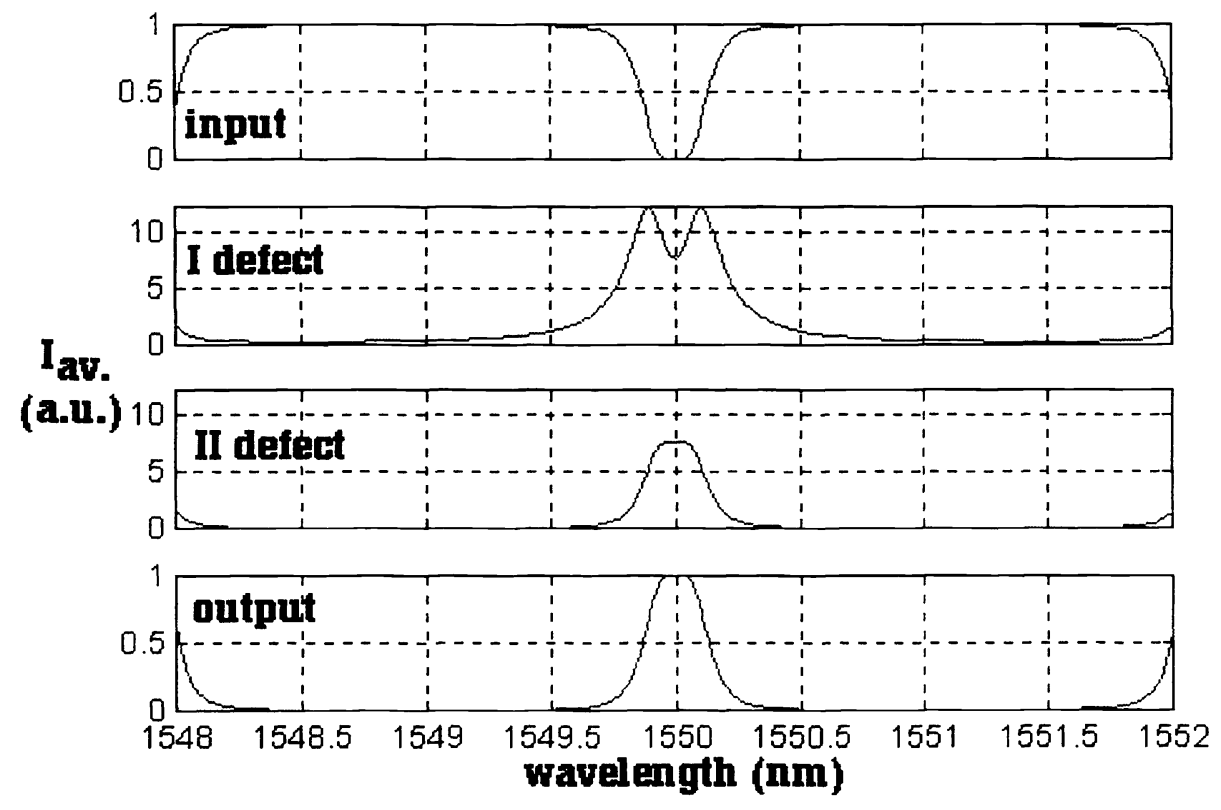

Figure 5: Optical intensity at the input, at the first defect, at the second defect and at the output (from top to bottom) for two weakly coupled defects. 


\section{III.2. Coupled PBG Cavities}

Recent studies on cascaded PBGs report the presence of coupling causing a splitting of the resonant transmission peak inside the stop-band [9]. A proposal for an optical demultiplexer based on this effect has also been reported [15]. For the simplest case of only two cascaded defects, when $L_{\text {int }}<2 L_{\text {ext }}$ (see fig. 1b), the central transmission peak at $\lambda_{B}$ splits in two symmetrical peaks. The separation is inversely proportional to $\mathrm{L}_{\text {int }}$. This result can be generalized to the case of more than two cascaded cavities where, under the stated condition, the central peaks splits in a number of peaks equal to the number of resonators.

By calculating the field distribution inside the device, we observe that the effect of splitting of the transmission peaks reflects the splitting in $\lambda$ of the resonant power intensity inside the defects. As a consequence, the condition required for forcing the cascaded resonators to have a resonant peak centered on a single wavelength $\left(\lambda_{B}\right)$ is $L_{\text {int }} \geq 2 L_{\text {ext }}$. This relation expresses a condition of weak coupling among the cascaded defects. Fig.5 shows $I_{a v}(\lambda)$ at the device input, at the output, and in the center of the defects of a PBG with two weakly-coupled defects. The plots refer to the case of $L_{\text {int }}=200 \mu \mathrm{m}, L_{\text {ext }}=100 \mu \mathrm{m}$, and

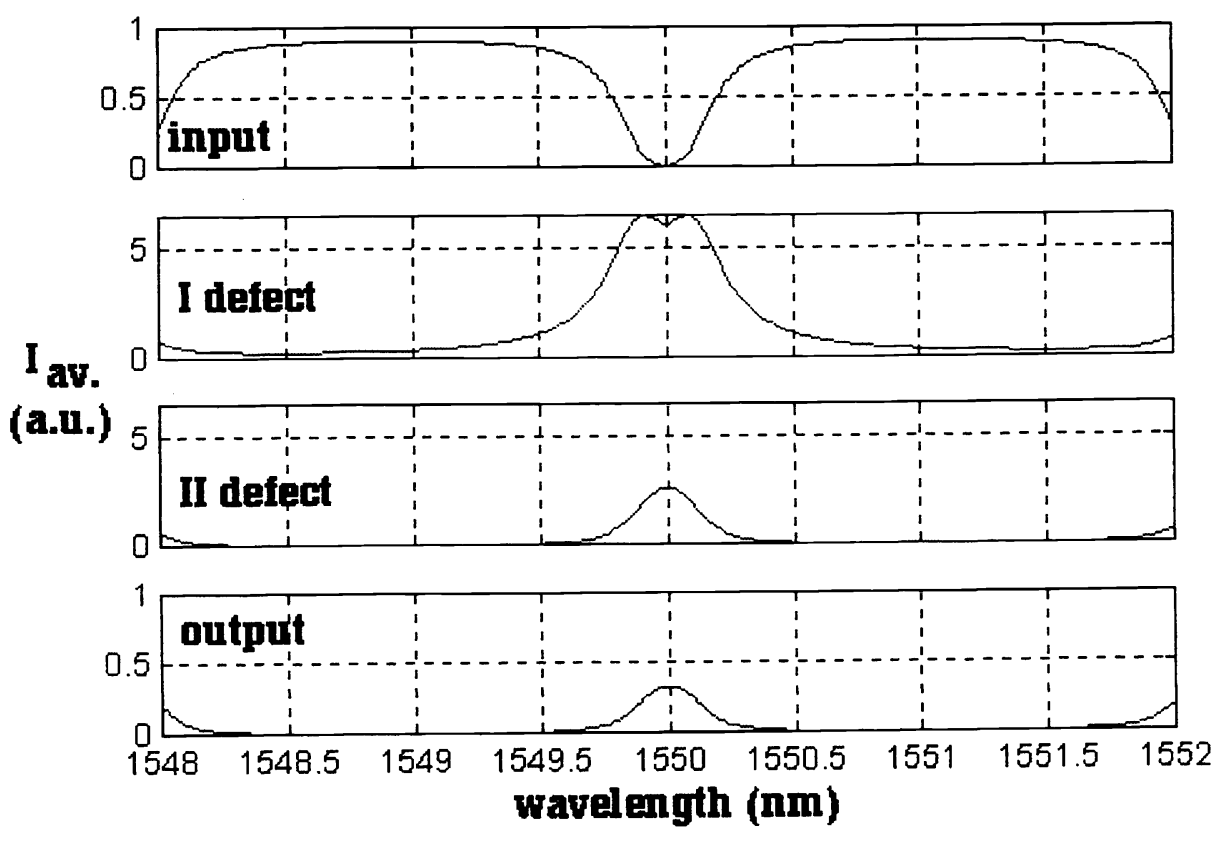

Figure 6: Optical intensity at the input, at the first defect, at the second defect and at the output (from top to bottom) for two weakly coupled defects. An effective material absorption of $\alpha=10 \mathrm{~cm}^{-1}$ is included. The intensity at the two different cascaded defects is no longer uniformly distributed. 


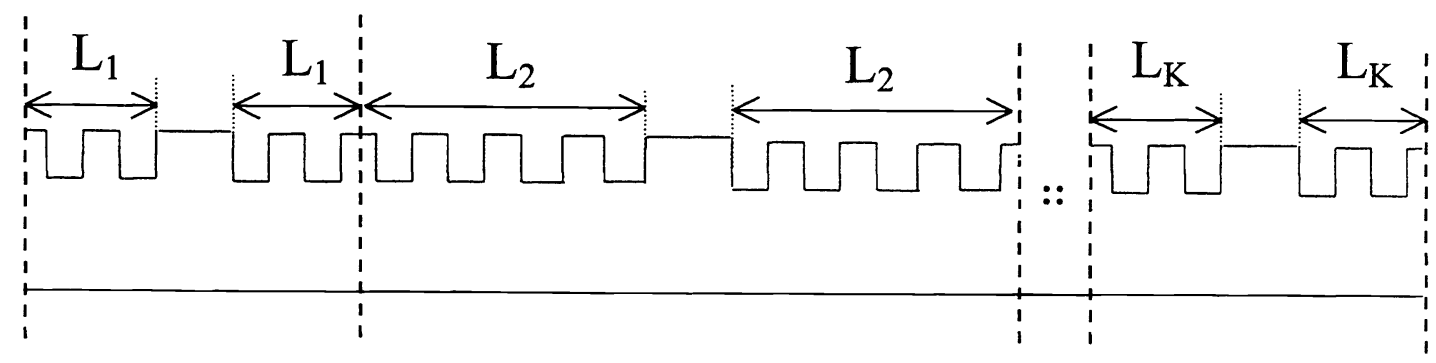

Figure 7: Schematic of cascaded structure with different grating length for each defect. The optical power can be uniformly distributed over the different defects.

$\Delta n=0.01$. Two general features can be extrapolated: 1) the profile of $I_{a v}(\lambda)$ in the first defect can differ from those in other as it must be consistent with the field intensity at the device input (i.e. reflectivity); 2) the value of $I_{a v}$ calculated in the central resonant wavelength $\left(I_{a v}\left(\lambda_{\mathrm{B}}\right)\right)$ is the same in all the defects as long as the condition $\mathrm{L}_{\mathrm{int}}=2 \mathrm{~L}_{\mathrm{ext}}$ is fulfilled, and the optical losses are negligible.

The analysis of Fig. 5 is repeated in Fig. 6 in the case where an effective absorption loss of $\alpha_{\text {eff. }}=10 \mathrm{~cm}^{-1}$ is present. With respect to the ideal case analyzed in fig.5, a progressive decaying of the
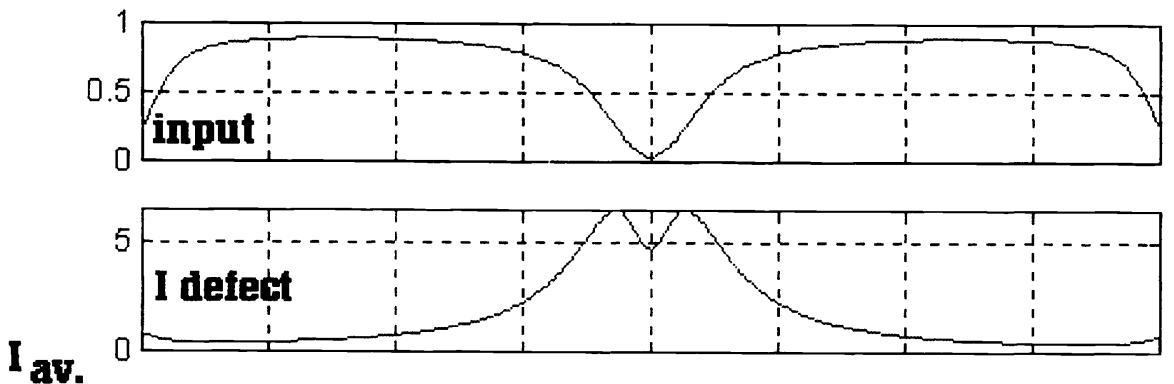

av.
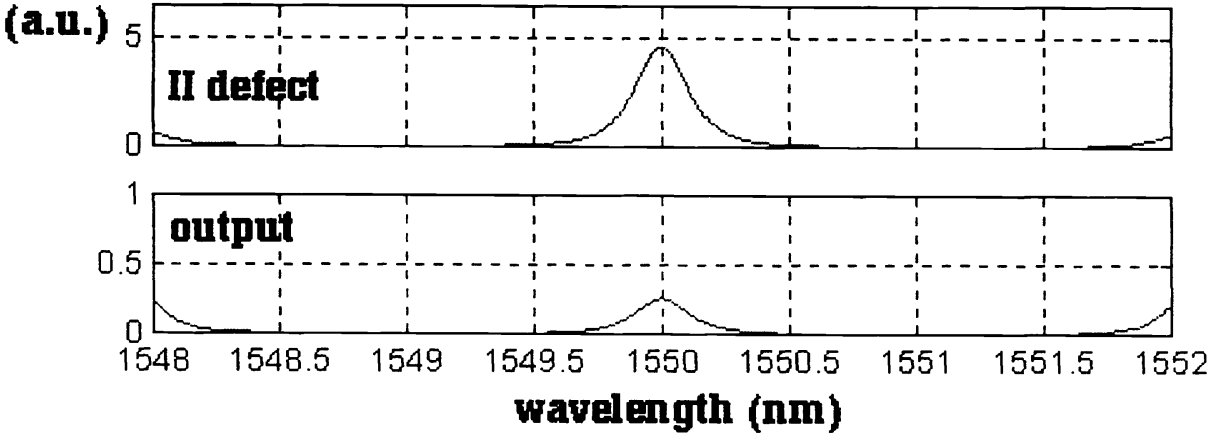

Figure 8: Intensity as a function of wavelength at the input, at the first defect, at the second defect and at the output of the structure. The structure is schematically described in figure 7 . The intensity is uniformly distributed over the two defects. An absorption of $\alpha=10 \mathrm{~cm}^{-1}$ is included. 
$I_{a v}\left(\lambda_{B}\right)$ along the structure due to the absorption attenuation effect is evident. Although the condition $\mathrm{L}_{\text {int }}=2$ $\mathrm{L}_{\mathrm{ext}}$ is satisfied, the average power intensity is no longer uniformly distributed among the different cascaded defects.

Resorting to the coupled-defects scheme of fig. 7 can solve this problem. In fact, in such a structure the weak-coupling condition is still satisfied and defects are forced to have a resonant peak centered at $\lambda_{\mathrm{B}}$. But the value of $I_{a v}\left(\lambda_{\mathrm{B}}\right)$ obtained in the $\mathrm{i}$-th $(\mathrm{i}=1, \ldots, \mathrm{K})$ defect increases dramatically with the length $\mathrm{L}_{\mathrm{i}}$, i.e., with the mirror reflectivity. Therefore, structures with $\mathrm{L}_{1}<\mathrm{L}_{2}<\cdots<\mathrm{L}_{\mathrm{K}}$, allow realization of devices (with nonzero absorption) where the power intensity is distributed uniformly among the different defects. In these devices, the attenuation is compensated by asymmetry in mirrors. In Fig.8, the same analysis of Fig. 6 is reported for a cascaded PBG with two weakly-coupled $\pi / 2$ defects, in the case of asymmetric mirror lengths $\left(\mathrm{L}_{1}=70 \mu \mathrm{m}, \mathrm{L}_{2}=130 \mu \mathrm{m}\right)$, and of a lossy structure $\left(\alpha_{\text {eff. }}=10 \mathrm{~cm}^{-1}\right) \Delta n=0.01$. The uniform $\operatorname{Iav}\left(\lambda_{\mathrm{B}}\right)$ obtained in the two defects is evident.

Our main interest in cascade-PBGs has been initially driven by ability to realize coupled-cavity distributed resonance. However, a more interesting result has emerged during our analysis: the possibility of the resonant-enhanced parallel detection of WDM optical signals. This promising feature is observed in

Iav.
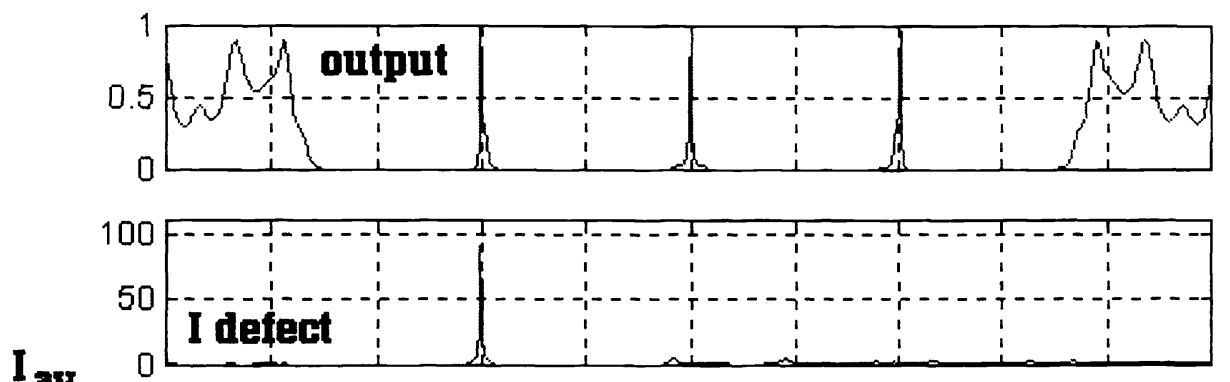

(a.u.) 1
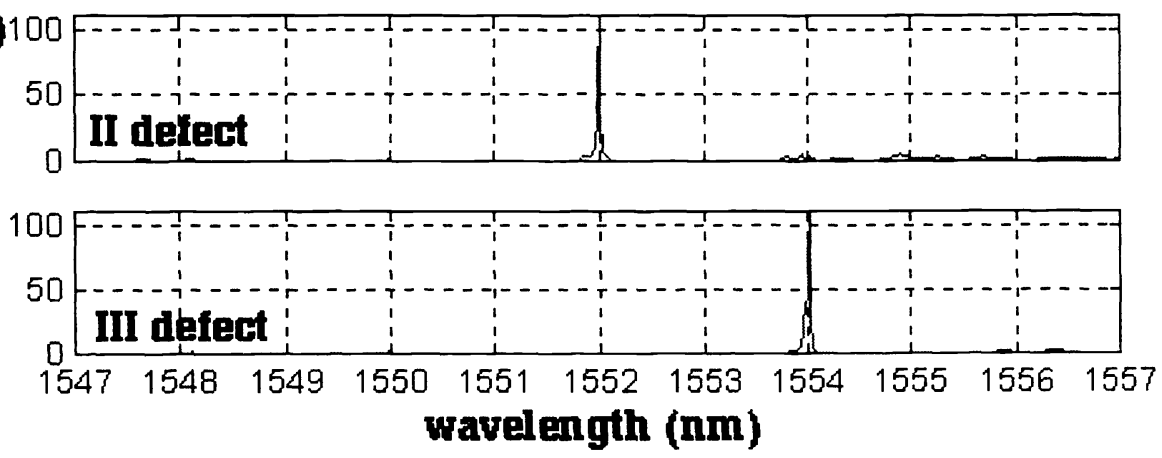

Figure 9: Intensity at the output of the structure and at the first second and third (Defect). The cavities are weakly coupled. The resonant wavelength for each cavity was designed with a $2 \mathrm{~nm}$ increment. The intensity at a resonant wavelength is located at the corresponding defect making this type of structure suitable for WDM application. 
the weakly-coupled distributed-resonance structures depicted in Fig. 7 by designing each section (for instance, the $\mathrm{i}$-th, with $\mathrm{i}=1, \ldots, \mathrm{K})$ so that its resonant wavelength $\left(\lambda_{\mathrm{B} i}\right)$ coincides with one of the particular wavelengths to be detected. In Fig. 9 we report the results of a PBG with 3 weakly-coupled defects. The sections are tuned to Bragg wavelengths of 1550,1552 , and $1554 \mathrm{~nm}$. The plots refer to a non-lossy structure with $\mathrm{L}_{1}=\mathrm{L}_{2}=\mathrm{L}_{3}=200 \mu \mathrm{m}$, and $\Delta n=0.01$. Each wavelength is selectively enhanced in a single defect. Such structures offer simultaneous demultiplexing and detection of WDM signals.

\section{CONCLUSIONS}

In summary, we have proposed a new type of resonant enhanced photodetectors based on onedimensional photonic bandgap. High quality factor and large FSR resonant cavities can be obtained by incorporating defect in the PBG structure.

Using the matrix formalism, we have studied transmission and reflection spectra, and the internal electric field distribution. A detailed analysis of weakly coupled cavity has been carried out. We have shown that the intensity distribution can be uniformly distributed between the cavities by changing the different length of the grating.

Finally, we have proposed a new type of photodetector for WDM applications. The structure consists of weakly couple cavities with different resonant frequencies. The electric field distribution calculations show that the electric field for a given resonant frequency is located only at the defect. Therefore, each defect can detect only its corresponding wavelength. This type of structure makes possible, resonant enhanced, parallel detection of individual channels in WDM optical communication systems.

\section{References}

[1] M. S. Ünlü, S. Strite, "Resonant cavity enhanced photonic devices, J. of Applied Phys., Vol. 78, No. 2, pp. $607-639,1995$.

[2] A. G. Dentai, et al., Electronics Lett., Vol.27, pp.2125-2126, 1991

[3] R. Kuchibhotla, et al., IEEE Photonics Tech. Lett., Vol.3, pp.354-356, 1991

[4]H. Temkin, T. P. Pearsall, J. C. Bean, and S. Luryi, " $\mathrm{Ge}_{x} \mathrm{Si}_{1-\mathrm{x}}$ strained-layer superlattice waveguide photodetectors operating near $1.3 \mu \mathrm{m}$ ", Appl. Phys. Lett., vol.48, No.15, pp.963-965, 1986.

[5] B. Jalali, L. Naval, A. F. J. Levi, "Si-based receivers for optical data links", J. of Lightwave Tech., Vol. 12, No. 6, pp. 930-935, 1994

[6] F. Y. Huang, K. Sakamoto, K. L. Wang, P. Trinh, B. Jalali, "Epitaxial SiGeC waveguide photodetector grown on Si substrate with response in the 1.3-1.55- $\mu \mathrm{m}$ wavelength range", IEEE Photonics Tech. Lett., Vol. 9, No. 2, pp.229-231, 1997 
[7] R. Kuchibhotla, J. C. Campbell, J. C. Bean, L. Peticolas, and R. Hull, " $\mathrm{Ge}_{0.1} \mathrm{Si}_{0.8} / \mathrm{Si}$ bragg reflector mirrors for optoelectronic device applications”, Appl. Phys. Lett., vol.62, No.18, pp.2215-2217, 1993.

[8] Guided-wave optoelectronics $-2^{\text {nd }}$ edition, T. Tamir ed., Springer-Verlag, Berlin, 1990

[9] H. A. Haus, and Y. Lai, "Theory of cascaded quarter wave shifted distributed feedback resonators", IEEE J. of Quantum El., Vol.28, N0.1, pp.205-213, 1992

[10] R. Zengerle, and O. Leminger, " Phase-shifted bragg-grating filters with improved transmission characteristics”, J. of Lightwave Tech., Vol.13, No.12, pp.2354-2358, 1995.

[11] J. N. Damask, V. V. Wong, J. Ferrera, H. I. Smith, H. A. Haus, "Highly coherent electron-beamwritten quarter-wave-shifted distributed bragg resonators for channel dropping filters, OFC '95 Technical Digest, pp.225-226, 1995

[12] H. F. Taylor, O. Eknoyan, C. S. Park, K. N. Choi, and K. Chang, "Traveling wave photodetectors", Proc. SPIE Vol.1217, pp.5963, 1990

[13] V. M. Hietala, G. A. Vawter, T. M. Brennan, and B. E. Hammons, “ Traveling wave photodetectors for higher-power, large-bandwidth applications”, IEEE Trans. Microwave Theory Tech., Vol.43, No.9, pp. 2291-2298, 1995

[14] L. Y. Lin, M. C. Wu, T. Itoh, T. A. Vang, R. E. Muller, D. L. Sivco, and A. Y. Cho, "Velocitymatched distributed photodetectors with high saturation power and large bandwidth", IEEE Photonics Tech. Lett., Vol.8, No.10, pp.1376-1378, 1996.

[15] G. P. Agrawal, and S. Radic, "Phase-shifted fiber bragg gratings and their applications for wavelength demultiplexing", IEEE Photonics Tech. Lett., vol.6, No.8, pp.995-997, 1994

[16] A. Yariv and P. Yeh, Optical waves in crystals, New York, Wiley, 1984

[17] G. Cocorullo, F. Della Corte, I. Rendina, and A. Cutolo, "New possibilities foe efficient silicon integrated electro-optical modulators”, Optics Communications, Vol.86, pp. 228-235, 1991

[18] M. A. Muriel, and A. Carballar, "Internal field distributions in fiber bragg gratings", IEEE Photonics Tech. Lett., Vol.9, No.7, pp. 955-957, 1997

[19] E. Yablonovitch, T.J. Gmitter, R.D. Meack, K.D. Brommer, A.M. Rappe, and J.D. Jeannopoulos, "Donor and acceptor modes in photonic band structure", Phys. Rev. Lett., 67, p. 3380, (1991).

[20] G. Cocorullo, F. G. Della Corte, and I. Rendina, "Thermo-optic effect exploitation in silicon microstructures", Proceedings of the $3^{\text {rd }}$ International Workshop on Thermal Investigations of ICs and Microstructures, Cannes (France), 1997, pp.235-241. 